\title{
SCHMIDT'S GAME, FRACTALS, AND NUMBERS NORMAL TO NO BASE
}

\author{
Ryan Broderick, Yann Bugeaud, Lior Fishman, \\ DMitry Kleinbock AND BARAK WeIsS
}

\begin{abstract}
Given $b>1$ and $y \in \mathbb{R} / \mathbb{Z}$, we consider the set of $x \in \mathbb{R}$ such that $y$ is not a limit point of the sequence $\left\{b^{n} x \bmod 1: n \in \mathbb{N}\right\}$. Such sets are known to have full Hausdorff dimension, and in many cases have been shown to have a stronger property of being winning in the sense of Schmidt. In this paper, by utilizing Schmidt games, we prove that these sets and their bi-Lipschitz images must intersect with 'sufficiently regular' fractals $K \subset \mathbb{R}$ (that is, supporting measures $\mu$ satisfying certain decay conditions). Furthermore, the intersection has full dimension in $K$ if $\mu$ satisfies a power law (this holds for example if $K$ is the middle third Cantor set). Thus it follows that the set of numbers in the middle third Cantor set which are normal to no base has dimension $\log 2 / \log 3$.
\end{abstract}

\section{Introduction}

Let $b \geq 2$ be an integer. A real number $x$ is said to be normal to base $b$ if, for every $n \in \mathbb{N}$, every block of $n$ digits from $\{0,1, \ldots, b-1\}$ occurs in the base- $b$ expansion of $x$ with asymptotic frequency $1 / b^{n}$. Equivalently, let $f_{b}$ be the self-map of $\mathbb{T} \stackrel{\text { def }}{=} \mathbb{R} / \mathbb{Z}$ given by $x \mapsto b x$, and denote by $\pi: x \rightarrow x \bmod 1$ the natural projection $\mathbb{R} \rightarrow \mathbb{T}$. Then $x$ is normal to base $b$ iff for any interval $I \subset \mathbb{T}$ with $b$-ary rational endpoints one has

$$
\lim _{n \rightarrow \infty} \frac{1}{n} \#\left\{0 \leq k \leq n-1: f_{b}^{k}(\pi(x)) \in I\right\}=\lambda(I),
$$

where $\lambda$ stands for Lebesgue measure. É. Borel established that $\lambda$-almost all numbers are normal to every integer base; clearly this is also a consequence of Birkhoff's Ergodic Theorem and the ergodicity of $\left(\mathbb{T}, \lambda, f_{b}\right)$.

Note that it is easy to exhibit many non-normal numbers in a given base $b$. For example, denote by $E_{b}$ the set of real numbers with a uniform upper bound on the number of consecutive zeroes in their base- $b$ expansion. Clearly those are not normal, and it is not hard to show that the Hausdorff dimension of $E_{b}$ is equal to 1. Furthermore, it was shown by W. Schmidt [26] that for any $b$ and any $0<\alpha<1 / 2$, the set $E_{b}$ is an $\alpha$-winning set of a game which later became known as Schmidt's game. This property implies full Hausdorff dimension but is considerably stronger; for example, the intersection of countably many $\alpha$-winning sets is also $\alpha$-winning (we describe the definition and features of Schmidt's game in $\S 3$ ). Thus it follows that the set of real numbers $x$ such that for each $b \in \mathbb{Z}_{\geq 2}$ their base- $b$ expansion does not contain more than $C=C(x, b)$ consecutive zeroes has full Hausdorff dimension. Obviously, such numbers are normal to no base.

Received by the editors September 25, 2009. Revision received on February 3, 2010. 
Now fix $y \in \mathbb{T}$ and a map $f: \mathbb{T} \rightarrow \mathbb{T}$, and, following notation introduced in [14], consider

$$
E(f, y) \stackrel{\text { def }}{=}\left\{x \in \mathbb{T}: y \notin \overline{\left\{f^{n}(x): n \in \mathbb{N}\right\}}\right\},
$$

the set of points with $f$-orbits staying away from $y$. For brevity we will write

$$
E(b, y)=\left\{x \in \mathbb{T}: y \notin \overline{\left\{f_{b}^{n}(x): n \in \mathbb{N}\right\}}\right\} .
$$

for $E\left(f_{b}, y\right)$. Obviously $E(b, 0)$ is a subset of $\pi\left(E_{b}\right)$ for any $b$. It is known that $\operatorname{dim}(E(b, y))=1$ for any $b$ and any $y \in \mathbb{T}$, see e.g. [29, 7]. Moreover, these sets ${ }^{1}$ have been recently proved by J. Tseng [28] to be $\alpha$-winning, where $\alpha$ is independent of $y$ but (quite badly) depends on $b$. In particular, it follows that for any bounded sequence $b_{1}, b_{2}, \ldots \in \mathbb{Z}_{\geq 2}$ and any $y_{1}, y_{2}, \ldots \in \mathbb{T}$, one has

$$
\operatorname{dim}\left(\bigcap_{k=1}^{\infty} E\left(b_{k}, y_{k}\right)\right)=1
$$

Another related result is that of S.G. Dani [6], who proved that for any $y \in \mathbb{Q} / \mathbb{Z}$ and any $b \in \mathbb{Z}_{\geq 2}$, the sets $E(b, y)$ are $\frac{1}{2}$-winning (in fact, his set-up is more general and involves semisimple endomorphisms of the $d$-dimensional torus). Consequently, (1.3) holds with no upper bound on $b_{k}$ as long as points $y_{k}$ are chosen to be rational (that is, pre-periodic for maps $f_{b}$ ).

The main purposes of the present note are to extend (1.3) by removing an upper bound ${ }^{2}$ on $b_{k}$, and to consider intersections with certain fractal subsets of $\mathbb{T}$ such as e.g. the middle third Cantor set. In fact it will be convenient to lift the problem from $\mathbb{T}$ to $\mathbb{R}$ and work with $\pi^{-1}(E(b, y))$; in other words, consider

$$
\tilde{E}(b, y) \stackrel{\text { def }}{=}\left\{x \in \mathbb{R}: y \notin \overline{\left\{\pi\left(b^{n} x\right): n \in \mathbb{N}\right\}}\right\}
$$

Clearly this set is periodic (with period 1); however we are going to study its intersections with (not necessarily periodic) subsets $K \subset \mathbb{R}$, for example, with their bi-Lipschitz images. Another advantage of switching from (1.2) to (1.4) is that the latter makes sense even when $b>1$ is not an integer ${ }^{3}$. This set-up has been extensively studied; for example A. Pollington proved in [24] that the intersection $\bigcap_{k=1}^{\infty} \tilde{E}\left(b_{k}, y_{k}\right)$ has Hausdorff dimension at least $1 / 2$ for any choices of $y_{k} \in \mathbb{T}$ and $b_{k}>1, k \in \mathbb{N}$. More generally, there are similar results with $\left(b^{n}\right)$ in (1.4) replaced by an arbitrary lacunary sequence $\mathcal{T}=\left(t_{n}\right)$ of positive real numbers (recall that $\mathcal{T}$ is called lacunary if $\left.\inf _{n \in \mathbb{N}} \frac{t_{n+1}}{t_{n}}>1\right)$. Namely, generalizing (1.4), fix $\mathcal{T}$ as above and a sequence $\mathcal{Y}=\left(y_{n}\right)$ of points in $\mathbb{T}$, and define

$$
\tilde{E}(\mathcal{T}, \mathcal{Y}) \stackrel{\text { def }}{=}\left\{x \in \mathbb{R}: \inf _{n \in \mathbb{N}} d\left(\pi\left(t_{n} x\right), y_{n}\right)>0\right\} .
$$

\footnotetext{
${ }^{1}$ The results of $[29,7,28]$, are more general, with $f_{b}$ replaced by an arbitrary sufficiently smooth expanding self-map of $\mathbb{T}$.

${ }^{2}$ After this paper was finished we learned of an alternative approach [10, 11] showing that sets $E(b, y)$ are $\frac{1}{4}$-winning for any $y \in \mathbb{T}$ and any $b \in \mathbb{Z}_{\geq 2}$; also, in a sequel [3] to the present paper it is explained that $\frac{1}{4}$ can be replaced by $\frac{1}{2}$.

${ }^{3}$ To make sense of $(1.2)$ when $b \notin \mathbb{Z}$ some efforts are required, see $\S 5.4$.
} 
Here and hereafter $d$ stands for the usual distance on $\mathbb{T}$ or $\mathbb{R}$. We will write $\tilde{E}(\mathcal{T}, y)$ when $\mathcal{Y}=(y)$ is a constant sequence, that is,

$$
\tilde{E}(\mathcal{T}, y) \stackrel{\text { def }}{=}\left\{x \in \mathbb{R}: y \notin \overline{\left\{\pi\left(t_{n} x\right): n \in \mathbb{N}\right\}}\right\} .
$$

It is a result of Pollington [25] and B. de Mathan [19] that the sets $\tilde{E}(\mathcal{T}, 0)$ have Hausdorff dimension 1 for any lacunary sequence $\mathcal{T}$; see also [4, Theorem 3] for a multi-dimensional generalization. Moreover, one can show, as mentioned by $\mathrm{N}$. Moshchevitin in [22], that those sets are $\frac{1}{2}$-winning.

Our main theorem extends the aforementioned results in several directions. We will allow arbitrary sequences $\mathcal{Y}$, and will study intersection of $\operatorname{sets} \tilde{E}(\mathcal{T}, \mathcal{Y})$ with certain fractals $K \subset \mathbb{R}$. Namely, if $K$ is a closed subset of the real line, following [12], we will play Schmidt's game on the metric space $K$ with the induced metric. We will say that a subset $S$ of $\mathbb{R}$ is $\alpha$-winning on $K$ if $S \cap K$ is an $\alpha$-winning set for the game played on $K$. See $\S 3$ for more detail. Further, in $\S 2$ we define and discuss so-called $(C, \gamma)$-absolutely decaying measures - a notion introduced in [15]. Here is our main result:

Theorem 1.1. Let $K$ be the support of a $(C, \gamma)$-absolutely decaying measure on $\mathbb{R}$, and let

$$
\alpha \leq \frac{1}{4}\left(\frac{1}{3 C}\right)^{\frac{1}{\gamma}}
$$

Then for every bi-Lipschitz map $\varphi: \mathbb{R} \rightarrow \mathbb{R}$, any sequence $\mathcal{Y}$ of points in $\mathbb{T}$, and any lacunary sequence $\mathcal{T}$, the set $\varphi(\tilde{E}(\mathcal{T}, \mathcal{Y}))$ is $\alpha$-winning on $K$.

We also show in $\S 3$ that when $K$ is as in the above theorem and $S$ is winning on $K$, one has $\operatorname{dim}(S \cap K) \geq \gamma$. Furthermore, $\operatorname{dim}(S \cap K)=\operatorname{dim}(K)$ if $\mu$ satisfies a power law. Consequently, in view of the countable intersection property of winning sets, for any choice of lacunary sequences $\mathcal{T}_{k}$, sequences $\mathcal{Y}_{k}$ of points in $\mathbb{T}$, and bi-Lipschitz maps $\varphi_{k}: \mathbb{R} \rightarrow \mathbb{R}$, one has

$$
\operatorname{dim}\left(K \cap \bigcap_{k=1}^{\infty} \varphi_{k}\left(\tilde{E}\left(\mathcal{T}_{k}, \mathcal{Y}_{k}\right)\right)\right) \geq \gamma,
$$

where $\gamma$ is as in Theorem 1.1 (see Corollary 4.2). Thus on any $K$ as above it is possible to find a set of positive Hausdorff dimension consisting of numbers which are normal to no base.

Another consequence of the generality of Theorem 1.1 is a possibility to consider orbits of affine expanding maps of the circle, that is,

$$
f_{b, c}: x \mapsto b x+c, \text { where } b \in \mathbb{Z}_{\geq 2} \text { and } c \in \mathbb{T} .
$$

It then follows that whenever $K, \alpha$ and $\varphi$ are as in Theorem 1.1 and $y \in \mathbb{T}$, the set $\varphi\left(\pi^{-1}\left(E\left(f_{b, c}, y\right)\right)\right)$ is $\alpha$-winning on $K$ (see Corollary 4.3$)$. In particular, $E\left(f_{b, c}, y\right)$ itself is $\alpha$-winning on any subset of $\mathbb{T}$ supporting a measure which can be lifted to a $(C, \gamma)$-absolutely decaying measure on $\mathbb{R}$.

Also, as is essentially proved in [12], a bi-Lipschitz image of the set

$$
\mathbf{B A} \stackrel{\text { def }}{=}\left\{x \in \mathbb{R}: \exists c=c(x)>0 \text { s. t. }\left|x-\frac{p}{q}\right|>\frac{c}{q^{2}} \forall(p, q) \in \mathbb{Z} \times \mathbb{N}\right\}
$$


of badly approximable numbers is also $\alpha$-winning on $K$ under the same assumptions on $K$ (see also [16,18]). We discuss this in $\S 4$ (see Theorem 4.1). Thus the intersection of the set in the left hand side of (1.6) with $\varphi(\mathbf{B A})$, where $\varphi: \mathbb{R} \rightarrow \mathbb{R}$ is bi-Lipschitz, will still have Hausdorff dimension at least $\gamma$. This significantly generalizes V. Jarník's [13] result on the full Hausdorff dimension of BA, as well as its strengthening by Schmidt [26]. Note that BA is a nonlinear analogue of $\tilde{E}(b, 0)$, with $f_{b}$ replaced by the Gauss map; this naturally raises a question of extending our results to more general self-maps of $\mathbb{T}$, see $\S 5.4$.

As a straightforward consequence of our results, we get

Corollary 1.2. Given $K \subset \mathbb{R}$ supporting an absolutely decaying measure $\mu$, the set of real numbers $x \in K$ that are badly approximable and such that, for every $b \geq 2$, their base-b expansion does not contain more than $C(x, b)$ consecutive identical digits, has positive Hausdorff dimension. In particular, if $\mu$ satisfies a power law (for example, if $K$ is the middle third Cantor set), then the dimension of this set is full.

The structure of the paper is as follows. In $\S 2$ we describe the class of absolutely decaying measures on $\mathbb{R}$, giving examples and highlighting the connections between absolute decay and other properties. In $\S 3$ we discuss Schmidt's game played on arbitrary metric spaces $X$, and then specialize to the case when $X=K$ is a subset of $\mathbb{R}$ supporting an absolutely decaying measure. Then in $\S 4$ we prove the main theorem. The last section is devoted to some extensions of our main result and further open questions.

\section{Absolutely decaying measures}

The next definition describes a property of measures first introduced in [15]. In this paper we only consider measures on the real line; however see $\S 5.5$ for a situation in higher dimensions. In what follows, we denote by $B(x, \rho)$ the closed ball in a metric space $(X, d)$ centered at $x$ of radius $\rho$,

$$
B(x, \rho) \stackrel{\text { def }}{=}\{y \in X: d(x, y) \leq \rho\} .
$$

Definition 2.1. Let $\mu$ be a locally finite Borel measure on $\mathbb{R}$, and let $C, \gamma>0$. We say that $\mu$ is $(C, \gamma)$-absolutely decaying if there exists $\rho_{0}>0$ such that for all $0<\rho \leq \rho_{0}$, $x \in \operatorname{supp} \mu, y \in \mathbb{R}$ and $\varepsilon>0$,

$$
\mu(B(x, \rho) \cap B(y, \varepsilon \rho))<C \varepsilon^{\gamma} \mu(B(x, \rho)) .
$$

We say $\mu$ is absolutely decaying if it is $(C, \gamma)$-absolutely decaying for some positive $C, \gamma$.

Many examples of measures satisfying this property are constructed ${ }^{4}$ in $[15,16]$. For example, limit measures of finite systems of contracting similarities $[15, \S 8]$ satisfying the open set condition and without a global fixed point are absolutely decaying. See also $[30,31,32,27]$ for other examples.

\footnotetext{
${ }^{4}$ The terminology in [15] is slightly different; there, $\mu$ is called absolutely decaying if $\mu$-almost every point has a neighborhood $U$ such that the restriction of $\mu$ to $U$ is $(C, \gamma)$-absolutely decaying for some $C, \gamma$; however in all examples considered in $[15,16]$ a stronger uniform property is in fact established.
} 
In what follows we highlight the connections between absolute decay and other conditions introduced earlier in the literature.

Definition 2.2. Let $\mu$ be a locally finite Borel measure on a metric space $X$. One says that $\mu$ is Federer (resp., efd) if there exists $\rho_{0}>0$ and $0<\varepsilon, \delta<1$ such that for every $0<\rho \leq \rho_{0}$ and for any $x \in \operatorname{supp} \mu$, the ratio

$$
\mu(B(x, \varepsilon \rho)) / \mu(B(x, \rho))
$$

is at least (resp., at most) $\delta$.

Federer property is usually referred to as 'doubling': see e.g. [20] for discussions and examples. The term 'efd' (an abbreviation for exponentially fast decay) was introduced by Urbanski; see [30, 32] for many examples and [33, 34] for other equivalent formulations. The next lemma provides another way to state these properties:

Lemma 2.3. Let $\mu$ be a locally finite Borel measure on a metric space $X$. Then $\mu$ is Federer (resp., efd) if and only if there exist $\rho_{0}>0$ and $c, \gamma>0$ such that for every $0<\rho \leq \rho_{0}, 0<\varepsilon<1$, and $x \in \operatorname{supp} \mu$, the ratio (2.3) is not less (resp., not greater) than $c \varepsilon^{\gamma}$.

Proof. The 'if' part is clear, one simply needs to choose $\varepsilon$ such that $c \varepsilon^{\gamma}<1$. Now suppose $\mu$ is Federer, and let $\varepsilon_{0}, \delta$ be such that

$$
\mu\left(B\left(x, \varepsilon_{0} \rho\right)\right) \geq \delta \mu(B(x, \rho))
$$

for every $0<\rho \leq \rho_{0}$ and $x \in \operatorname{supp} \mu$. We are going to put $c=\delta$ and $\gamma=\frac{\log \delta}{\log \varepsilon_{0}}$. Take $0<\varepsilon<1$, and let $n$ be the largest integer such that $\varepsilon \leq \varepsilon_{0}^{n}$. Then

$$
c \varepsilon^{\gamma}=\delta \varepsilon^{\frac{\log \delta}{\log \varepsilon_{0}}}=\delta \delta^{\frac{\log \varepsilon}{\log \varepsilon_{0}}} \leq \delta^{n+1} .
$$

Hence

$$
c \varepsilon^{\gamma} \mu(B(x, \rho)) \leq \delta^{n+1} \mu(B(x, \rho)) \underset{(2.4) \text { applied } n \text { times }}{\leq} \mu\left(B\left(x, \varepsilon_{0}^{n+1} \rho\right)\right),
$$

which, in view of the definition of $n$, implies $\mu(B(x, \varepsilon \rho)) \geq c \varepsilon^{\gamma} \mu(B(x, \rho))$. Similarly, from the fact that $\mu\left(B\left(x, \varepsilon_{0} \rho\right)\right) \leq \delta \mu(B(x, \rho))$ for every $0<\rho \leq \rho_{0}$ and $x \in \operatorname{supp} \mu$ one can deduce the inequality

$$
\mu(B(x, \varepsilon \rho)) \leq c \varepsilon^{\gamma} \mu(B(x, \rho))
$$

for every $x, \rho$ and $\varepsilon$, with $c=1 / \delta$ and $\gamma=\frac{\log \delta}{\log \varepsilon_{0}}$.

Now we can produce an alternative description of absolutely decaying measures on $\mathbb{R}:$

Proposition 2.4. Let $\mu$ be a locally finite Borel measure on $\mathbb{R}$. Then $\mu$ is absolutely decaying if and only if it is Federer and efd.

The 'if' part is due to Urbanski, see [32, Lemma 7.1]; we include a proof to make the paper self-contained. 
Proof. Let $\mu$ be $(C, \gamma)$-absolutely decaying, and let $\rho_{0}$ be as in Definition 2.1. Taking $x=y$ and $c=C$ in (2.2) readily implies (2.5), i.e. the efd property. To show Federer, take $0<\rho \leq \rho_{0}$ and $x \in \operatorname{supp} \mu$, and let $\varepsilon<1 / 4$ satisfy $C \varepsilon^{\gamma}<1 / 2$. Choose $y_{1}$ and $y_{2}$ to be the two distinct points satisfying $\left|x-y_{i}\right|=(1-\varepsilon) \rho, i=1,2$. It clearly follows from Definition 2.1 that $\mu$ is non-atomic; thus we can write

$$
\mu(B(x, \rho))=\mu\left(B(x, \rho) \cap B\left(y_{1}, \varepsilon \rho\right)\right)+\mu(B(x,(1-2 \varepsilon) \rho))+\mu\left(B(x, \rho) \cap B\left(y_{2}, \varepsilon \rho\right)\right) .
$$

Therefore, by $(2.2)$,

$$
\mu(B(x, \rho)) \leq \mu(B(x,(1-2 \varepsilon) \rho))+2 C \varepsilon^{\gamma} \mu(B(x, \rho)) .
$$

Setting $\varepsilon_{0}=1-2 \varepsilon$ and $\delta=1-2 C \varepsilon^{\gamma}$ we get (2.4).

Conversely, suppose that $\mu$ is both Federer and efd. In view of Lemma 2.3, for some $\rho_{0}>0$ and $c_{1}, c_{2}, \gamma_{1}, \gamma_{2}>0$ one has

$$
c_{1} \varepsilon^{\gamma_{1}} \mu(B(x, \rho)) \leq \mu(B(x, \varepsilon \rho)) \leq c_{2} \varepsilon^{\gamma_{2}} \mu(B(x, \rho))
$$

for all $0<\rho \leq \rho_{0}, x \in \operatorname{supp} \mu$ and $0<\varepsilon<1$. Now take $\rho<\rho_{0} / 3$ and $y \in B(x, \rho)$. If $\mu(B(x, \rho) \cap B(y, \varepsilon \rho))=0$, we are done. Otherwise, there exists $y^{\prime} \in \operatorname{supp} \mu \cap$ $B(y, \varepsilon \rho) \cap B(x, \rho)$. Then

$$
\begin{gathered}
\mu(B(x, \rho) \cap B(y, \varepsilon \rho)) \leq \mu\left(B\left(y^{\prime}, 2 \varepsilon \rho\right)\right) \leq c_{2} \varepsilon^{\gamma_{2}} \mu\left(B\left(y^{\prime}, 2 \rho\right)\right) \\
\leq c_{2} \varepsilon^{\gamma_{2}} \mu(B(x, 3 \rho)) \leq c_{2} c_{1}^{-1} 3^{\gamma_{1}} \varepsilon^{\gamma_{2}} \mu(B(x, \rho)),
\end{gathered}
$$

which gives (2.2) with $C=c_{2} c_{1}^{-1} 3^{\gamma_{1}}$ and $\gamma=\gamma_{2}$.

In particular, suppose that $\mu$ satisfies a power law, i.e. there exist positive $\gamma, k_{1}$, $k_{2}, \rho_{0}$ such that for every $x \in \operatorname{supp} \mu$ and $0<\rho<\rho_{0}$ one has

$$
k_{1} \rho^{\gamma} \leq \mu(B(x, \rho)) \leq k_{2} \rho^{\gamma} ;
$$

then $\mu$ is clearly efd and Federer, hence absolutely decaying. However there exist examples of absolutely decaying measures without a power law, see [16, Example 7.5]. Also, recall that the lower pointwise dimension of $\mu$ at $x$ is defined as

$$
\underline{d}_{\mu}(x) \stackrel{\text { def }}{=} \liminf _{\rho \rightarrow 0} \frac{\log \mu(B(x, \rho))}{\log \rho},
$$

and, for an open $U$ with $\mu(U)>0$ let

$$
\underline{d}_{\mu}(U) \stackrel{\text { def }}{=} \inf _{x \in \operatorname{supp} \mu \cap U} \underline{d}_{\mu}(x) .
$$

Then it is known, see e.g. [9, Proposition 4.9], that (2.6) constitutes a lower bound for the Hausdorff dimension of supp $\mu \cap U$ (this bound is sharp when $\mu$ satisfies a power law). It is easy to see that $\underline{d}_{\mu}(x) \geq \gamma$ for every $x \in \operatorname{supp} \mu$ whenever $\mu$ is $(C, \gamma)$-absolutely decaying: indeed, let $\rho_{0}$ be as in Definition 2.1 and take $\rho<\rho_{0}$ and $x \in \operatorname{supp} \mu$; then, letting $\varepsilon=\frac{\rho}{\rho_{0}}$, one has

$$
\mu(B(x, \rho)) \leq C\left(\frac{\rho}{\rho_{0}}\right)^{\gamma} \mu\left(B\left(x, \rho_{0}\right)\right),
$$

thus, for $\rho<1$,

$$
\frac{\log \mu(B(x, \rho))}{\log \rho} \geq \gamma+\frac{\log C-\gamma \log \rho_{0}+\log \mu\left(B\left(x, \rho_{0}\right)\right)}{\log \rho},
$$


and the claim follows.

In the next section we will show that sets supporting absolutely decaying measures on $\mathbb{R}$ work very well as playing fields for Schmidt's game. The aforementioned lower estimate for $\underline{d}_{\mu}(x)$ will be used to provide a lower bound for the Hausdorff dimension of winning sets of the game.

\section{Schmidt's game}

In this section we describe the game, first introduced by Schmidt in [26]. Let $(X, d)$ be a complete metric space. Consider $\Omega \stackrel{\text { def }}{=} X \times \mathbb{R}_{+}$, and define a partial ordering

$$
\left(x_{2}, \rho_{2}\right) \leq_{s}\left(x_{1}, \rho_{1}\right) \text { if } \rho_{2}+d\left(x_{1}, x_{2}\right) \leq \rho_{1} .
$$

We associate to each pair $(x, \rho)$ a ball in $(X, d)$ via the 'ball' function $B(\cdot)$ as in (2.1). Note that $\left(x_{2}, \rho_{2}\right) \leq_{s}\left(x_{1}, \rho_{1}\right)$ clearly implies (but is not necessarily implied by) $B\left(x_{2}, \rho_{2}\right) \subset B\left(x_{1}, \rho_{1}\right)$. However the two conditions are equivalent when $X$ is a Euclidean space.

Schmidt's game is played by two players, whom, following a notation used in [17], we will call ${ }^{5}$ Alice and Bob. The two players are equipped with parameters $\alpha$ and $\beta$ respectively, satisfying $0<\alpha, \beta<1$. Choose a subset $S$ of $X$ (a target set). The game starts with Bob picking $x_{1} \in X$ and $\rho>0$, hence specifying a pair $\omega_{1}=\left(x_{1}, \rho\right)$. Alice and Bob then take turns choosing $\omega_{k}^{\prime}=\left(x_{k}^{\prime}, \rho_{k}^{\prime}\right) \leq_{s} \omega_{k}$ and $\omega_{k+1}=\left(x_{k+1}, \rho_{k+1}\right) \leq_{s} \omega_{k}^{\prime}$ respectively satisfying

$$
\rho_{k}^{\prime}=\alpha \rho_{k} \text { and } \rho_{k+1}=\beta \rho_{k}^{\prime} .
$$

As the game is played on a complete metric space and the diameters of the nested balls

$$
B\left(\omega_{1}\right) \supset \ldots \supset B\left(\omega_{k}\right) \supset B\left(\omega_{k}^{\prime}\right) \supset \ldots
$$

tend to zero as $k \rightarrow \infty$, the intersection of these balls is a point $x_{\infty} \in X$. Call Alice the winner if $x_{\infty} \in S$. Otherwise Bob is declared the winner. A strategy consists of specifications for a player's choices of centers for his or her balls given the opponent's previous moves.

If for certain $\alpha, \beta$ and a target set $S$ Alice has a winning strategy, i.e., a strategy for winning the game regardless of how well Bob plays, we say that $S$ is an $(\alpha, \beta)$ winning set. If $S$ and $\alpha$ are such that $S$ is an $(\alpha, \beta)$-winning set for all $\beta$ in $(0,1)$, we say that $S$ is an $\alpha$-winning set. Call a set winning if such an $\alpha$ exists.

Intuitively one expects winning sets to be large. Indeed, every such set is clearly dense in $X$; moreover, under some additional assumptions on the metric space winning sets can be proved to have positive, and even full, Hausdorff dimension. For example, the fact that a winning subset of $\mathbb{R}^{n}$ has Hausdorff dimension $n$ is due to Schmidt [26, Corollary 2]. Another useful result of Schmidt [26, Theorem 2] states that the intersection of countably many $\alpha$-winning sets is $\alpha$-winning.

Schmidt himself used the machinery of the game he invented to prove that certain subsets of $\mathbb{R}$ or $\mathbb{R}^{n}$ are winning, and hence have full Hausdorff dimension. For example, he showed [26, Theorem 3] that BA is $\alpha$-winning for any $0<\alpha \leq 1 / 2$. The same conclusion, according to $[26, \S 8]$, holds for the sets $E_{b}$ defined in the introduction.

\footnotetext{
${ }^{5}$ The players were referred to as 'white' and 'black' by Schmidt, and as $A$ and $B$ in some subsequent literature; a suggestion to use the Alice/Bob nomenclature is due to Andrei Zelevinsky.
} 
Now let $K$ be a closed subset of $X$. We will say that a subset $S$ of $X$ is $(\alpha, \beta)$ winning on $K$ (resp., $\alpha$-winning on $K$, winning on $K$ ) if $S \cap K$ is $(\alpha, \beta)$-winning (resp., $\alpha$-winning, winning) for Schmidt's game played on the metric space $K$ with the metric induced from $(X, d)$. In the present paper we let $X=\mathbb{R}$ and take $K$ to be the support of an absolutely decaying measure. In other words, since the metric is induced, playing the game on $K$ amounts to choosing balls in $\mathbb{R}$ according to the rules of a game played on $\mathbb{R}$, but with an additional constraint that the centers of all the balls lie in $K$.

It turns out, as was observed in [12], that the decay property (2.2) is very helpful for playing Schmidt's game on $K$. Moreover, as demonstrated by the following proposition proved in [17], the decay conditions are important for estimating the Hausdorff dimension of winning sets:

Proposition 3.1. [17, Proposition 5.1] Let $K$ be the support of a Federer measure $\mu$ on a metric space $X$, and let $S$ be winning on $K$. Then for any open $U \subset X$ with $\mu(U)>0$ one has

$$
\operatorname{dim}(S \cap K \cap U) \geq \underline{d}_{\mu}(U) .
$$

In particular, in the above proposition one can replace $\underline{d}_{\mu}(U)$ with $\gamma$ if $\mu$ is $(C, \gamma)$ absolutely decaying. Note that this generalizes estimates for the Hausdorff dimension of winning sets due to Schmidt [26] for $\mu$ being Lebesgue measure on $\mathbb{R}^{n}$, and to Fishman $[12, \S 5]$ for measures satisfying a power law.

The next lemma is another example of the absolute decay of a measure being helpful for playing Schmidt's game on its support:

Lemma 3.2. Let $K$ be the support of a $(C, \gamma)$-absolutely decaying measure on $\mathbb{R}$, and let $\alpha$ be as in (1.5). Then for every $0<\rho<\rho_{0}, x_{1} \in K$ and $y_{1}, \ldots, y_{N} \in \mathbb{R}$, there exists $x_{1}^{\prime} \in K$ with

$$
B\left(x_{1}^{\prime}, \alpha \rho\right) \subset B\left(x_{1}, \rho\right)
$$

and, for at least half of the points $y_{i}$,

$$
d\left(B\left(x_{1}^{\prime}, \alpha \rho\right), y_{i}\right)>\alpha \rho .
$$

Proof. If $B\left(x_{1}, 2 \alpha \rho\right)$ contains not more than half of the points $y_{i}$, then clearly we can take $x_{1}^{\prime}=x_{1}$. Otherwise, $B\left(x_{1}, 2 \alpha \rho\right)$ contains at least half of the points $y_{i}$. Let $x_{0}$ and $x_{2}$ be the endpoints of $B\left(x_{1}, \rho\right)$. By $(2.2)$

$$
\mu\left(B\left(x_{i}, 4 \alpha \rho\right)\right)<C(4 \alpha)^{\gamma} \mu\left(B\left(x_{1}, \rho\right)\right) \underset{(1.5)}{<} \frac{1}{3} \mu\left(B\left(x_{1}, \rho\right)\right),
$$

for $i=0,1,2$, so there is a point $x_{1}^{\prime} \in K$ which is not in $B\left(x_{i}, 4 \alpha \rho\right)$ for $i=0,1,2$, and hence satisfies both (3.2) and (3.3) for all $y_{i}$ contained in $B\left(x_{1}, 2 \alpha \rho\right)$.

We note that (3.2) in particular implies that $\left(x_{1}^{\prime}, \alpha \rho\right) \leq_{s}\left(x_{1}, \rho\right)$; thus it would be a valid choice of Alice in an $(\alpha, \beta)$-game played on $K$ in response to $B\left(x_{1}, \rho\right)$ chosen by Bob. Therefore the above lemma can be used to construct a winning strategy for Alice choosing balls which stay away from some prescribed sets of 'bad' points $y_{1}, \ldots, y_{N}$. This idea is motivated by the proof of Lemma 1 in [23].

Furthermore, the above lemma immediately implies 
Corollary 3.3. Let $K$ be the support of a $(C, \gamma)$-absolutely decaying measure on $\mathbb{R}$, let $\alpha$ be as in (1.5), let $S \subset \mathbb{R}$ be $\alpha$-winning on $K$, and let $S^{\prime} \subset S$ be countable. Then $S \backslash S^{\prime}$ is also $\alpha$-winning on $K$.

Proof. In view of the countable intersection property, it suffices to show that $\mathbb{R} \backslash\{y\}$ is $(\alpha, \beta)$-winning on $K$ for any $y$ and any $\beta$. We let Alice play arbitrarily until the radius of a ball chosen by Bob is not greater than $\rho_{0}$. Then apply Lemma 3.2 with $N=1$ and $y_{1}=y$, which yields a ball not containing $y$. Afterwards she can keep playing arbitrarily, winning the game.

We note that such a property is demonstrated in [26, Lemma 14] for games played on a Banach space of positive dimension.

\section{Proofs}

Proof of Theorem 1.1. Let $\alpha$ be as in (1.5) and let $0<\beta<1$. Suppose $K$ supports a $(C, \gamma)$-absolutely decaying measure, $\varphi: \mathbb{R} \rightarrow \mathbb{R}$ is bi-Lipschitz, $\mathcal{T}=\left(t_{n}\right)$ is a sequence of positive reals satisfying

$$
\inf _{n} \frac{t_{n+1}}{t_{n}}=M>1,
$$

and $\mathcal{Y}=\left(y_{n}\right)$ is a sequence of points in $\mathcal{T}$. Our goal is to specify a strategy for Alice allowing to zoom in on $\varphi(\tilde{E}(\mathcal{T}, \mathcal{Y})) \cap K$.

Choose $N$ large enough so that

$$
(\alpha \beta)^{-r} \leq M^{N}, \text { where } r \stackrel{\text { def }}{=}\left\lfloor\log _{2} N\right\rfloor+1 .
$$

Here and hereafter $\lfloor\cdot\rfloor$ denotes the integer part.

Note that without loss of generality one can replace the sequence $\mathcal{T}$ with its tail $\mathcal{T}^{\prime} \stackrel{\text { def }}{=}\left(t_{n}: n \geq n_{0}\right)$; indeed, it is easy to see that

$$
\tilde{E}(\mathcal{T}, \mathcal{Y}) \backslash \tilde{E}\left(\mathcal{T}^{\prime}, \mathcal{Y}^{\prime}\right),
$$

where $\mathcal{Y}^{\prime} \stackrel{\text { def }}{=}\left(y_{n}: n \geq n_{0}\right)$, is at most countable; therefore the claim follows from Corollary 3.3. Consequently, one can assume that ${ }^{6} t_{n}>1$ for all $n$.

Let $L$ be a bi-Lipschitz constant for $\varphi$; in other words,

$$
\frac{1}{L} \leq \frac{|\varphi(x)-\varphi(y)|}{|x-y|} \leq L \quad \forall x \neq y \in \mathbb{R} .
$$

The game begins with Bob choosing $\left(x_{1}, \rho^{\prime}\right) \in \Omega=K \times \mathbb{R}_{+}$. Let $k_{0}$ be the minimal positive integer satisfying

$$
\rho \stackrel{\text { def }}{=}(\alpha \beta)^{k_{0}-1} \rho^{\prime}<\min \left(\frac{1}{2 L}(\alpha \beta)^{-r+1}, \rho_{0}\right),
$$

where $\rho_{0}$ is as in Definition 2.1. Alice will play arbitrarily until her $k_{0}$ th turn. Then $\omega_{k_{0}}=\left(x_{2}, \rho\right)$ for some $x_{2} \in K$. Reindexing, set $\omega_{1}=\omega_{k_{0}}$. Let

$$
c \stackrel{\text { def }}{=} \frac{\rho}{L}(\alpha \beta)^{3 r} \text {. }
$$

\footnotetext{
${ }^{6}$ The same argument shows that the assumption of the lacunarity of $\mathcal{T}$ in Theorem 1.1 can be weakened to eventual lacunarity, that is, to $\liminf _{n \rightarrow \infty} \frac{t_{n+1}}{t_{n}}>1$.
} 
For an arbitrary $k \in \mathbb{N}$, define

$$
I_{k} \stackrel{\text { def }}{=}\left\{n \in \mathbb{N}:(\alpha \beta)^{-r(k-1)} \leq t_{n}<(\alpha \beta)^{-r k}\right\} ;
$$

note that $\# I_{k} \leq N$ in view of (4.1) and (4.2).

Our goal now is to describe Alice's strategy for choosing $\omega_{i}^{\prime} \in \Omega, i \in \mathbb{N}$, to ensure that for any $k \in \mathbb{N}$,

$$
d\left(\pi\left(t_{n} \varphi^{-1}(x)\right), y_{n}\right) \geq c \text { whenever } x \in B\left(\omega_{r(k+2)-1}^{\prime}\right) \text { and } n \in I_{k} .
$$

Then if we let

$$
x_{\infty} \stackrel{\text { def }}{=} \bigcap_{i} B\left(\omega_{i}^{\prime}\right)=\bigcap_{k} B\left(\omega_{r(k+2)-1}^{\prime}\right),
$$

which is clearly an element of $K$, we will have $\varphi^{-1}\left(x_{\infty}\right) \in \tilde{E}(\mathcal{T}, \mathcal{Y})$; in other words, (4.5) enforces that $x_{\infty} \in \varphi(\tilde{E}(\mathcal{T}, \mathcal{Y})) \cap K$, as required.

To achieve (4.5), Alice may choose $\omega_{i}^{\prime}$ arbitrarily for $i<2 r$. Now fix $k \in \mathbb{N}$ and observe that whenever $n \in I_{k}$ and $m_{1} \neq m_{2} \in \mathbb{Z}$, one has

$$
\left|\frac{y_{n}+m_{1}}{t_{n}}-\frac{y_{n}+m_{2}}{t_{n}}\right| \geq t_{n}^{-1}>(\alpha \beta)^{r k},
$$

so, by (4.3),

$$
\left|\varphi\left(\frac{y_{n}+m_{1}}{t_{n}}\right)-\varphi\left(\frac{y_{n}+m_{2}}{t_{n}}\right)\right|>\frac{1}{L}(\alpha \beta)^{r k} .
$$

Because of (4.4), the diameter of $B\left(\omega_{r(k+1)}\right)$ is

$$
2(\alpha \beta)^{r(k+1)-1} \rho<\frac{1}{L}(\alpha \beta)^{r k},
$$

so by (4.6) the set

$$
Z \stackrel{\text { def }}{=}\left\{\varphi\left(\frac{y_{n}+m}{t_{n}}\right): m \in \mathbb{Z}, n \in I_{k}\right\}
$$

has at most $N$ elements in $B\left(\omega_{r(k+1)}\right)$. Applying Lemma $3.2 r$ times, Alice can choose $\omega_{r(k+1)}^{\prime}, \ldots, \omega_{r(k+2)-1}^{\prime} \in \Omega$ in such a way that

$$
d\left(B\left(\omega_{r(k+2)-1}^{\prime}\right), Z\right) \geq(\alpha \beta)^{r(k+2)} \rho .
$$

Therefore, again by (4.3), for any $x \in B\left(\omega_{r(k+2)-1}^{\prime}\right), m \in \mathbb{Z}$ and $n \in I_{k}$ one has

$$
\left|t_{n} \varphi^{-1}(x)-\left(y_{n}+m\right)\right| \geq \frac{t_{n}}{L}\left|x-\varphi\left(\frac{y_{n}+m}{t_{n}}\right)\right| \geq \frac{t_{n}}{L}(\alpha \beta)^{r(k+2)} \rho \geq \frac{\rho}{L}(\alpha \beta)^{3 r}=c,
$$

which implies (4.5).

Recall that it was shown in [26] that BA is a winning subset of $\mathbb{R}$. In [12], this set, and its nonsingular affine images, was shown to be $\alpha$-winning on the support of any $(C, \gamma)$-absolutely decaying measure on $\mathbb{R}$, where $\alpha$ depends only on $C$ and $\gamma$. In what follows we prove a slight generalization of this result for bi-Lipschitz images. The technique used is similar to the one used in the proof of the main theorem. We include it for the sake of completeness. 
Theorem 4.1. Let $K$ be the support of a $(C, \gamma)$-absolutely decaying measure on $\mathbb{R}$, and let $\alpha$ be as in (1.5). Then for every bi-Lipschitz map $\varphi: \mathbb{R} \rightarrow \mathbb{R}$, the set $\varphi(\mathbf{B A})$ is $\alpha$-winning on $K$.

Proof. Again, take an arbitrary $0<\beta<1$, and let $L$ be as in (4.3). Let $R=(\alpha \beta)^{-\frac{1}{2}}$. The game begins with Bob choosing $\left(x_{1}, \rho^{\prime}\right) \in \Omega$. Let $k_{0}$ be the minimal positive integer satisfying

$$
(\alpha \beta)^{k_{0}-1} \rho^{\prime}<\min \left(\frac{\alpha \beta}{2 L}, \rho_{0}\right),
$$

where $\rho_{0}$ is as in Definition 2.1, and denote $\rho \stackrel{\text { def }}{=}(\alpha \beta)^{k_{0}-1} \rho^{\prime}$. Alice will play arbitrarily until her $k_{0}$ th turn. Then $\omega_{k_{0}}=\left(x_{2}, \rho\right)$ for some $x_{2} \in K$. Reindexing, set $\omega_{1}=\omega_{k_{0}}$. Let $c=\frac{R^{2} \alpha \rho}{L}$.

Fix an arbitrary $k \in \mathbb{N}$. We will describe Alice's strategy for choosing $\omega_{k}^{\prime}$ such that

$$
\left|\varphi^{-1}(x)-\frac{p}{q}\right|>\frac{c}{q^{2}} \text { for all } x \in B\left(\omega_{k}^{\prime}\right), R^{k-1} \leq q<R^{k} .
$$

Clearly the existence of such strategy implies that she can play so that $\bigcap_{k} B\left(\omega_{k}^{\prime}\right)$ lies in $K \cap \varphi(\mathbf{B A})$.

Note that for any distinct $\frac{p_{1}}{q_{1}}, \frac{p_{2}}{q_{2}} \in \mathbb{R}$ with $R^{k-1} \leq q_{1}, q_{2}<R^{k}$,

$$
\left|\frac{p_{1}}{q_{1}}-\frac{p_{2}}{q_{2}}\right|=\left|\frac{p_{1} q_{2}-p_{2} q_{1}}{q_{1} q_{2}}\right|>\frac{1}{R^{2 k}} .
$$

Hence, $\left|\varphi\left(\frac{p_{1}}{q_{1}}\right)-\varphi\left(\frac{p_{2}}{q_{2}}\right)\right| \geq \frac{1}{L} R^{-2 k}$. But

$$
\operatorname{diam}\left(B\left(\omega_{k}\right)\right) \leq 2 \rho(\alpha \beta)^{k-1} \underset{(4.7)}{<} \frac{1}{L} R^{-2 k},
$$

so $B\left(\omega_{k}\right)$ contains at most one point $\varphi\left(\frac{p}{q}\right)$ with $R^{k-1} \leq q<R^{k}$. In view of Lemma 3.2 , where we put $N=1$, Alice can choose $\omega_{k}^{\prime} \in \Omega$ such that, for every $x \in B\left(\omega_{k}^{\prime}\right)$ and $(p, q) \in \mathbb{Z} \times \mathbb{N}$ with $R^{k-1} \leq q<R^{k}$, one has

$$
\left|x-\varphi\left(\frac{p}{q}\right)\right|>\alpha \rho(\alpha \beta)^{k}=\alpha \rho R^{-2 k}>\frac{R^{2} \alpha \rho}{q^{2}} .
$$

Again by (4.3), we obtain

$$
\left|\varphi^{-1}(x)-\frac{p}{q}\right|>\frac{R^{2} \alpha \rho}{L q^{2}}=\frac{c}{q^{2}},
$$

and (4.8) is established.

As an immediate consequence of Proposition 3.1 and the countable intersection property of winning sets, we obtain the following

Corollary 4.2. Let $K$ be the support of a $(C, \gamma)$-absolutely decaying measure on $\mathbb{R}$, and let $\alpha$ be as in (1.5). Then given lacunary sequences $\mathcal{T}_{k}$, sequences $\mathcal{Y}_{k} \in \mathbb{T}$, biLipschitz maps $\varphi_{k}, \psi_{k}: \mathbb{R} \rightarrow \mathbb{R}$, and an open set $U \subset \mathbb{R}$ with $U \cap K \neq \varnothing$, one has

$$
\operatorname{dim}\left(\bigcap_{k=1}^{\infty} K \cap U \cap \varphi_{k}(\mathbf{B A}) \cap \psi_{k}\left(\tilde{E}\left(\mathcal{T}_{k}, \mathcal{Y}_{k}\right)\right)\right) \geq \gamma .
$$


In particular we can have $\gamma=\operatorname{dim}(K)$ when the measure satisfies a power law (e.g. when $K$ is equal to $\mathbb{R}$ or to the middle third Cantor set).

We conclude the section with an application of Theorem 1.1 to affine expanding maps $f_{b, c}$ as defined in (1.7):

Corollary 4.3. Let $K$ be the support of a $(C, \gamma)$-absolutely decaying measure on $\mathbb{R}$, and let $\alpha$ be as in (1.5). Then for every bi-Lipschitz map $\varphi: \mathbb{R} \rightarrow \mathbb{R}, b \in \mathbb{Z}_{\geq 2}$ and $c, y \in \mathbb{T}$, the set $\varphi\left(\pi^{-1}\left(E\left(f_{b, c}, y\right)\right)\right)$ is $\alpha$-winning on $K$.

Proof. Since $f_{b, c}$ is a composition of $f_{b}$ with an isometry of $\mathbb{T}$, it is easy to construct a sequence of points $\mathcal{Y}=\left(y_{n}\right)$ of $\mathbb{T}$ such that, with $\mathcal{T}=\left(b^{n}\right)$, one has $x \in \tilde{E}(\mathcal{T}, \mathcal{Y})$ if and only if $\pi(x) \in E\left(f_{b, c}, y\right)$.

\section{Applications, related results and further questions}

5.1. Trajectories avoiding intervals. Recently a quantitative modification of Schmidt's proof of abundance of numbers normal to no base was introduced in the work of R. Akhunzhanov. To describe it, let us define

$$
\hat{E}(b, A)=\bigcap_{y \in A} \tilde{E}(b, y)=\left\{x \in \mathbb{R}: A \cap \overline{\left\{\pi\left(b^{n} x\right): n \in \mathbb{N}\right\}}=\varnothing\right\}
$$

for a subset $A$ of $\mathbb{T}$. Clearly when $A=B(0, \delta)$ is a $\delta$-neighborhood of 0 in $\mathbb{T}$, every number $x \in \hat{E}(b, A)$ has a uniform (depending on $\delta$ ) upper bound on the number of consecutive zeros in the $b$-ary expansion. It is easy to see that whenever $A$ contains an interval, $\hat{E}(b, A)$ is nowhere dense and has positive Hausdorff codimension. Nevertheless it was proved in $[1,2]$ that for any $\varepsilon>0$ and any integer $b \geq 2$ there exists a positive (explicitly constructed) $\delta=\delta_{b, \varepsilon}$ such that the set

$$
\bigcap_{b \in \mathbb{Z}_{\geq 2}} \hat{E}\left(b, B\left(0, \delta_{b, \varepsilon}\right)\right)
$$

has Hausdorff dimension at least $1-\varepsilon$. The proof is based on Schmidt's game, namely on so-called $(\alpha, \beta, \rho)$-winning sets of the game. This technique readily extends to playing on supports of absolutely decaying measures. Namely, one can show that given $C, \gamma, \varepsilon>0$ and integer $b \geq 2$, there exists $\delta=\delta_{C, \gamma, b, \varepsilon}$ such that

$$
\operatorname{dim}\left(\bigcap_{b \in \mathbb{Z}_{\geq 2}} K \cap \hat{E}\left(b, B\left(0, \delta_{C, \gamma, b, \varepsilon}\right)\right)\right)>\gamma-\varepsilon
$$

whenever $K$ supports a $(C, \gamma)$-absolutely decaying measure. Details will be described elsewhere.

5.2. Are these sets null? It is not hard to construct examples of absolutely decaying measures $\mu$ such that $K=\operatorname{supp} \mu$ lies entirely inside a set of the form $\tilde{E}(b, y)$ for some $b \in \mathbb{Z}_{\geq 2}$, or inside the set of badly approximable numbers. However in many cases, under some additional assumptions on $\mu$ one can show that those sets, proved to be winning on $K$ in the present paper, have measure zero. For example, it is proved in [5] that almost all $x$ in the middle third Cantor set, with respect to the coin-flipping measure, are normal to base $b$ whenever $b$ is not a power of 3 . And in a recent work 
[8] of M. Einsiedler, U. Shapira and the third-named author it is established that $\mu(\mathbf{B A})=0$ whenever $\mu$ is $f_{b}$-invariant for some $b \in \mathbb{Z}_{\geq 2}$ and has positive dimension. It seems interesting to ask for general conditions on a measure on $\mathbb{R}$, possibly stated in terms of invariance under some dynamical system, which guarantee that whenever $y \in \mathbb{T}$, sets $\tilde{E}(b, y)$ for a fixed $b \geq 2$ have measure zero.

5.3. Strong winning sets. In a recent preprint [21] C. McMullen introduced a modification of Schmidt's game, where condition (3.1) is replaced by

$$
\rho_{k}^{\prime} \geq \alpha \rho_{k} \text { and } \rho_{k+1} \geq \beta \rho_{k}^{\prime},
$$

and $S \subset X$ is said to be $(\alpha, \beta)$-strong winning if Alice has a winning strategy in the game dened by (5.1). Analogously, we define $\alpha$-strong winning and strong winning sets. It is straightforward to verify that $(\alpha, \beta)$-strong winning implies $(\alpha, \beta)$-winning, and that a countable intersection of $\alpha$-strong winning sets is $\alpha$-strong winning. Furthermore, this class has stronger invariance properties, e.g. it is proved in [21] that strong winning subsets of $\mathbb{R}^{n}$ are preserved by quasisymmetric homeomorphisms. McMullen notes that many examples of winning sets arising naturally in dynamics and Diophantine approximation seem to also be strong winning. The sets considered in this paper are no exception: it is not hard to modify our proofs to show that, under the assumptions of Theorems 1.1 and 4.1 , the sets $\tilde{E}(\mathcal{T}, \mathcal{Y})$ and BA are $\alpha$-strong winning on $K$.

5.4. More general self-maps of $\mathbb{T}$. It would be interesting to unify Theorems 1.1 and 4.1 by describing a class of maps $f: \mathbb{T} \rightarrow \mathbb{T}$ for which one can prove sets of the form $E(f, y)$ to be winning on $K$ whenever $K \subset \mathbb{T}$ supports an absolutely decaying measure. An important special case is a map $f$ given by multiplication by $b$ when $b>1$ is not an integer; that is, constructed by identifying $\mathbb{T}$ with $[0,1)$ and defining $f(x)=b x \bmod 1$. With this definition, the set (1.4) does not coincide with the $\pi$ preimage of $E(f, y)$, and the methods of the present paper do not seem to yield any information. Some results along these lines have been obtained recently in $[10,11]$.

5.5. A generalization to higher dimensions. The method developed in the present paper has been extended in [3] to a multi-dimensional set-up, that is, with a lacunary sequence of real numbers acting on $\mathbb{R}$ replaced by a sequence of $m \times n$ matrices, whose operator norms form a lacunary sequence, acting on $\mathbb{R}^{n}$. This, among other things, generalizes a result of Dani [6] on orbits of toral endomorphisms. A higher-dimensional analogue of Theorem 1.1 can be established for absolutely decaying measures on $\mathbb{R}^{n}$. Note that the definition of absolutely decaying measures on $\mathbb{R}^{n}[15]$ is the same as Definition 2.1 but with balls $B(y, \varepsilon \rho)$ being replaced by $\varepsilon \rho$ neighborhoods of affine hyperplanes. Also, Proposition 2.4 does not extend to $n>1$, that is, absolute decay does not imply Federer, and a combination of efd and Federer does not imply absolute decay.

\section{Acknowledegments}

Yann Bugeaud would like to thank Ben Gurion University at Beer-Sheva, where part of this work has been done. This research was supported in part by NSF grant DMS-0801064, ISF grant 584/04 and BSF grant 2004149. 


\section{References}

[1] R. K. Akhunzhanov, On nonnormal numbers, Mat. Zametki 72 (2002), 150-152 (in Russian); translation in Math. Notes 72 (2002), 135-137.

[2] - On the distribution modulo 1 of exponential sequences, Mat. Zametki 76 (2004), 163171 (in Russian); translation in Math. Notes 76 (2004), 153-160.

[3] R. Broderick, L. Fishman and D. Kleinbock, Schmidt's game, fractals, and orbits of toral endomorphisms, Preprint, arXiv:1001.0318.

[4] Y. Bugeaud, S. Harrap, S. Kristensen and S. Velani, On shrinking targets for $\mathbb{Z}^{m}$ actions on tori, To appear in Mathematika.

[5] J.W.S. Cassels, On a problem of Steinhaus about normal numbers, Colloq. Math. 7 (1959), 95-101.

[6] S.G. Dani, On orbits of endomorphisms of tori and the Schmidt game, Ergod. Theory Dynam. Systems 8 (1988), 523-529.

[7] D. Dolgopyat, Bounded orbits of Anosov flows, Duke Math. J. 87 (1997), no. 1, 87-114.

[8] M. Einsiedler, L. Fishman and U. Shapira, Diophantine approximation on fractals, Preprint, arXiv:0908.2350.

[9] K. Falconer, Fractal geometry. Mathematical foundations and applications, John Wiley \& Sons, Inc., Hoboken, NJ, 2003.

[10] D. Färm, Simultaneously Non-dense Orbits Under Different Expanding Maps, Preprint, arXiv: $0904.4365 \mathrm{v} 1$.

[11] D. Färm, T. Persson and J. Schmeling, Dimension of Countable Intersections of Some Sets Arising in Expansions in Non-Integer Bases, To appear in Fundamenta Math.

[12] L. Fishman, Schmidt's game on fractals, Israel J. Math. 171 (2009), no. 1, 77-92.

[13] V. Jarník, Zur metrischen Theorie der Diophantischen Approximationen, Prace Math-fiz. 362. Heft (1928).

[14] D. Kleinbock, Nondense orbits of flows on homogeneous spaces, Ergodic Theory Dynam. Systems 18 (1998), 373-396.

[15] D. Kleinbock, E. Lindenstrauss and B. Weiss, On fractal measures and diophantine approximation, Selecta Math. 10 (2004), 479-523.

[16] D. Kleinbock and B. Weiss, Badly approximable vectors on fractals, Israel J. Math. 149 (2005), $137-170$

[17] Modified Schmidt games and Diophantine approximation with weights, Advances in Mathematics 223 (2010), 1276-1298.

[18] S. Kristensen, R. Thorn, S.L. Velani, Diophantine approximation and badly approximable sets, Advances in Math. 203 (2006), 132-169.

[19] B. de Mathan, Numbers contravening a condition in density modulo 1, Acta Math. Acad. Sci. Hungar. 36 (1980), 237-241.

[20] D. Mauldin and M. Urbanski, The doubling property of conformal measures of infinite iterated function systems, J. Number Th. 102 (2003), 23-40.

[21] C. McMullen, Winning sets, quasiconformal maps and Diophantine approximation, Preprint (2010).

[22] N.G. Moshchevitin, Sublacunary sequences and winning sets, Mat. Zametki 77 (2005), no. 6, 803-813 (in Russian); translation in Math. Notes 78 (2005), no. 4, 592-596.

[23] _ A note on badly approximable affine forms and winning sets, Preprint (2008), arXiv: 0812.3998v2.

[24] A.D. Pollington, On nowhere dense $\Theta$-sets, Groupe de travail d'analyse ultramtrique. 10 (19821983), no. 2, Exp. No. 22, 2 p.

[25] _ On the density of sequence $\left\{\eta_{k} \xi\right\}$, Illinois J. Math. 23 (1979), no. 4, 511-515.

[26] W.M. Schmidt, On badly approximable numbers and certain games, Trans. A.M.S. 123 (1966), $27-50$.

[27] B. Stratmann and M. Urbanski, Diophantine extremality of the Patterson measure, Math. Proc. Cambridge Phil. Soc. 140 (2006), 297-304.

[28] J. Tseng, Schmidt games and Markov partitions, Nonlinearity 22 (2009), no. 3, 525-543.

[29] M. Urbanski, The Hausdorff dimension of the set of points with non-dense orbit under a hyperbolic dynamical system, Nonlinearity 4 (1991), 385-397. 
[30] _ Diophantine approximation of self-conformal measures, J. Number Th. 110 (2005), $219-235$

[31] _ Diophantine approximation of conformal measures of one-dimensional iterated function systems, Compositio Math. 141 (2005), 869-886.

[32] _ Finer Diophantine and regularity properties of 1-dimensional parabolic IFS, Real Anal. Exchange 31 (2005/06), no. 1, 143-163.

[33] W.A. Veech, Measures supported on the set of uniquely ergodic directions of an arbitrary holomorphic 1-form, Ergodic Theory Dynam. Systems 19 (1999), 1093-1109.

[34] B. Weiss, Almost no points on a Cantor set are very well approximable, Proc. R. Soc. Lond. A 457 (2001), 949-952.

Department of Mathematics, Brandeis University, Waltham MA 02454-9110, USA

E-mail address: ryanb@brandeis.edu

Département de Mathématiques, Université de Strasbourg, 67084 Strasbourg, France

E-mail address: bugeaud@math.u-strasbg.fr

Department of Mathematics, Brandeis University, Waltham MA 02454-9110, USA

E-mail address: lfishman@brandeis.edu

Department of Mathematics, Brandeis University, Waltham MA 02454-9110, USA

E-mail address: kleinboc@brandeis.edu

Department of Mathematics, Ben Gurion University, Be'er Sheva, Israel 84105

E-mail address: barakw@math.bgu.ac.il 